\title{
EDITORIAL
}

\section{Plugging (publicising) to prevent mucous plugging}

\author{
P-R. Burgel* and J.A. Nadel ${ }^{\#, \varphi_{9}+}$
}

$\mathbf{M}$ ucus has long been known to exist in airways, but it is only relatively recently that mucous hypersecretion has been widely recognised to play major roles in the pathogenesis of chronic obstructive lung diseases, including asthma, chronic obstructive pulmonary disease and cystic fibrosis. Airway mucus is a complex mixture of liquids and proteins. Important components are gel-forming mucins, which are high-molecular-weight glycoproteins whose unusual viscoelastic properties permit the gel to interact with epithelial cilia, thus promoting clearance of deposited foreign materials. Airway mucins are reviewed in detail elsewhere [1]. Owing to its clinical importance and the limited effectiveness of current therapies [2, 3], mucous hypersecretion is an appealing target for novel therapies.

How has mucus evolved as an important source of host defence in the airways, and what are the roles of mucous secretion in chronic obstructive airway diseases? When fish migrated from water to land, lungs replaced gills for respiratory gas exchange. Placement of the lungs adjacent to the heart, deep within the thoracic cavity, added the requirement of an air tube to transport inspired air to the lungs. However, inspired air also contains microbes and other irritants, which are deposited on the airway epithelial surface, causing inflammation and invading the host. In response, the host has developed defensive actions, which normally destroy and clear the invading organisms. Among the defensive host responses to inhaled invaders, secreted airway mucins normally play major protective roles. The recruited mucins entrap the invaders, which are then cleared by cough and mucociliary clearance [4]. In healthy individuals, mucins in the airways are sparse but sufficient to accomplish entrapment and clearance of invaders efficiently and with minimal symptoms. However, in chronic inflammatory airway diseases, such as asthma, exaggerated mucin production occurs, leading to symptoms and airway obstruction. In the major conducting airways, hypersecretion leads to cough and sputum production. In small peripheral airways, because of their geometry, mucous hypersecretion may lead to plugging of the airway lumen. In fatal asthma, mucous hypersecretion is conspicuous and is believed to play a major role as a cause of death [5-8].

Various investigators have emphasised the importance of obstruction of small airways (mucous plugging). It should be

\footnotetext{
*Service de Pneumologie, Hôpital Cochin, Assistance Publique - Hôpitaux de Paris, Université Paris-Descartes, Paris, France. " Cardiovascular Research Institute, and Depts of "Medicine and +Physiology, University of California San Francisco, San Francisco, CA, USA.

CORRESPONDENCE: P-R. Burgel, Hôpital Cochin, 27, rue du Faubourg Saint Jacques, 75679 Paris Cedex 14, France. E-mail: pierre-regis.burgel@cch.aphp.fr
}

noted that, because of the localisation of plugged lumens in small airways, clinical recognition of mucous plugs has been problematic, and peripheral airway obstruction has remained a relatively silent zone of the lungs, not readily identifiable physiologically, by radiological examination or by bronchoscopic study. The diagnosis of airway plugging remains a challenge for investigators!

Recently, mucous hypersecretion became recognised as a major clinical problem in chronic airway diseases, and studies of mucins have intensified. First, mucin genes were cloned, providing tools for further mucin research. Since invading organisms, allergens, cigarette smoke and other irritating particulates are deposited on the epithelial surface, investigators sought signalling mechanisms that permitted the host to intercept the invaders.

When an invader is deposited on the surface of the airway epithelium, a cascade of events has been shown to occur, culminating in the activation of a receptor on the epithelial surface, the epidermal growth factor receptor (EGFR), and resulting in mucin production [9]. Subsequently, a wide variety of stimuli have been found to cause mucin production and other defensive responses via activation of EGFR [10]. In the original study, which reported that activation of EGFR by an EGFR ligand resulted in mucin production, it was also reported that ovalbumin-induced mucin production in rats was inhibited by a selective EGFR tyrosine kinase inhibitor, implicating an EGFR cascade in allergen-induced mucin production [9]. Subsequent studies have described a series of signals involved in an epithelial surface cascade, inducing EGFR activation and different outputs (e.g. interleukin-8 and antibacterial peptides) by multiple stimuli (e.g. Pseudomonas aeruginosa, viruses, lipopolysaccharide, cigarette smoke, allergens, neutrophil elastase and oxidants) [10]. The signalling pathway includes activation of a surface receptor (e.g. the Tolllike receptor (TLR)), activation of protein kinase $C$, generation of reactive oxygen species, activation of a surface matrix metalloprotease (MMP), often tumour necrosis factor- $\alpha$-converting enzyme (TACE), which cleaves membrane-bound EGFR ligands, which bind to and activate EGFR, resulting in the production of a variety of proteins and glycoproteins [1].

In 2009, ZHu et al. [11] reported that the major and minor groups of rhinovirus (RV) induced mucin production, which involves a novel TLR3-EGFR-dependent pathway. Their studies implicated TLR3 in the signalling cascade, and these authors suggested that the TLR antiviral defence machinery and the phosphatidylinositol-3'-kinase proliferation/repair of the EGFR pathway might play an important role in virusinduced airway disease exacerbations. This article did not 
indicate a mechanism for the special (exaggerated) effects in asthma. In another interesting study by BARTLETT et al. [12], ovalbumin-induced allergic airway disease was augmented by infection with RV-1B, but the mechanism of augmentation was not described.

In the present issue of the European Respiratory Journal, Hewson et al. [13] studied RV induction of mucin 5 subtypes A and C (MUC5AC), initially in asthmatic volunteers, and reported that experimental infection with RV-16 led to MUC5AC induction in proportion to virus load. Then they infected a lung cancer cell line (NCI-H292) with RV-16 and showed that RV infection caused significant induction of mucin. MUC5AC mRNA was increased dose-dependently between 8 and $48 \mathrm{~h}$ after infection (peak $24 \mathrm{~h}$ ). RV-induced mucin expression was inhibited by a selective EGFR inhibitor and suppressed by an inhibitor of mitogen-activated protein kinase kinase (MEK) activation. The MEK inhibitor blocked the mucin response 3-24 h after infection. They reported that RV infection induced MEK phosphorylation at 3-24 h (fig. 4c), and an inhibitor of Sp1 binding resulted in inhibition of mucin expression (fig. 4d). An inhibitor of inhibitor of nuclear factor- $\mathrm{\kappa B}$ kinase subunit $\beta$ (fig. 5b) inhibited mucin expression.

Then they examined whether or not RV infection induced the release of transforming growth factor- $\alpha$ (TGF- $\alpha$ ) and whether this

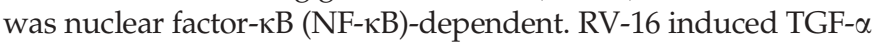
release and was significantly blocked by NF- $\kappa B$ inhibition (fig. 6b). The authors concluded that the signalling pathway of RV induction of NF- $\kappa$ B activated MMPs and a subsequent EGFRMEK/extracellular signal-regulated kinase pathway leading to MUC5AC synthesis and secretion. The most novel and stimulating aspect of this work is the proposed description of a virusinduced signalling pathway leading to mucin production.

Generally, in prior studies, multiple stimuli have been reported as causing activation of a sequence of surface signalling molecules and resulting in EGFR activation, including signalling by the receptor relevant to the stimulus (often a TLR), followed by the release of reactive oxygen species, activation of an MMP, often TACE, resulting in the cleavage of EGFR proligand, which then binds to and activates EGFR. This sequence leads via a series of downstream molecules (e.g. Ras, Raf and mitogen-activated protein kinase) and via a promoter to stimulation of production of new protein (e.g. mucins). In the present article by HewsON et al. [13], the authors suggest that the stimulus (RV) enters the cell and directly activates NF- $\mathrm{B}$, which then stimulates an MMP, and thus induces a continued cascade, including proligand cleavage, release of soluble EGFR ligand and activation of EGFR. This is a novel concept and would rearrange the sequence and timing of the signalling cascade (fig. 8 of [13]). In order to validate this pathway, the issues of timing are critical. For example, when does EGFR phosphorylation occur? In figure 3b, RV-16 activates EGFR at 8-12 h. In prior publications, investigators have found EGFR responses within minutes remaining for $\sim 0.5-1 \mathrm{~h}$ [10]. In HEwsON et al. [13], EGFR phosphorylation is not shown prior to $4 \mathrm{~h}$. Is there earlier EGFR phosphorylation? Curiously, mucin mRNA is reported at $8 \mathrm{~h}$, perhaps before EGFR phosphorylation is reported. Thus, in future studies, the issue of timing and analysis of the signalling based on time are critical for determining how this signalling pathway functions. Furthermore, mechanisms of RV recognition on the epithelial surface, entry of RV within the epithelial cell and its relation to EGFR activation require additional studies.

Cells survive by modulating their activities over time. In this regard, investigators have struggled to develop systems biology for the prediction of complex events. However, systems biology analysis shows a great deal of difficulty in building time into systems! Here, we suggest that a careful dissection of signalling pathways over time may continue to be useful in discovering biological mechanisms.

Currently, the clinical importance of mucous hypersecretion in airway disease is recognised, and investigators have increased studies of the basic mechanisms, including mucin production and the mechanisms underlying mucous hypersecretion. The clinical importance of peripheral mucous plugging and the difficulty in diagnosing these lesions require novel approaches to both diagnosis and therapy. It is necessary to determine the mechanisms that cause viral infections to trigger asthma exacerbations and how these exacerbations can be prevented or effectively treated. Since nonsmall lung cancer cells, which were used in the study of Hewson et al. [13], may exhibit substantially different signalling mechanisms from asthmatics, the use of primary culture of epithelial cells obtained from asthmatic subjects may provide new insights. These are pressing issues that urgently require further investigation. The present effects of deteriorated health and the serious financial impact of viral infections in asthmatics presently mandate a high priority for future research in clarifying the mechanisms involved in mucous hypersecretion.

Here is one final comment for pulmonary investigators. The pulmonary specialty was late in its development. However, because of the importance of lungs and airways in inflammatory, immune and malignant diseases, their location adjacent to the atmosphere and their ease of access, it is predicted that growth in pulmonary research will continue to accelerate! We are plugging for it!

\section{STATEMENT OF INTEREST}

A statement of interest for J.A. Nadel can be found at www.erj. ersjournals.com/site/misc/statements.xhtl

\section{REFERENCES}

1 Nadel JA. Airway epithelial mucins and mucous hypersecretion. In: Murray JF, Nadel JA, eds. Textbook of Respiratory Medicine. 5th Edn. Philadelphia, Elsevier, 2010; pp. 226-235.

2 King M, Rubin BK. Pharmacological approaches to discovery and development of new mucolytic agents. Adv Drug Deliv Rev 2002 54: 1475-1490.

3 Curran DR, Cohn L. Advances in mucous cell metaplasia: a plug for mucus as a therapeutic focus in chronic airway disease. Am J Respir Cell Mol Biol 2010; 42: 268-275.

4 O'Riordan T, Smaldone GC. Aerosol deposition and clearance. In: Murray JF, Nadel JA, eds. Textbook of Respiratory Medicine. 5th Edn. Philadelphia, Elsevier, 2010; pp. 236-254.

5 Cardell BS, Pearson RSB. Death in asthmatics. Thorax 1959; 14: 341-352.

6 Aikawa T, Shimura S, Sasaki H, et al. Marked goblet cell hyperplasia with mucus accumulation in the airways of patients who died of severe acute asthma attack. Chest 1992; 101: 916-921.

7 Shimura S, Andoh Y, Haraguchi M, et al. Continuity of airway goblet cells and intraluminal mucus in the airways of patients with bronchial asthma. Eur Respir J 1996; 9: 1395-1401. 
8 Groneberg DA, Eynott PR, Lim S, et al. Expression of respiratory mucins in fatal status asthmaticus and mild asthma. Histopathology 2002; 40: 367-373.

9 Takeyama K, Dabbagh K, Lee HM, et al. Epidermal growth factor system regulates mucin production in airways. Proc Natl Acad Sci USA 1999; 96: 3081-3086.

10 Burgel PR, Nadel JA. Epidermal growth factor receptor-mediated innate immune responses and their roles in airway diseases. Eur Respir J 2008; 32: 1068-1081.
11 Zhu L, Lee PK, Lee WM, et al. Rhinovirus-induced major airway mucin production involves a novel TLR3-EGFR-dependent pathway. Am J Respir Cell Mol Biol 2009; 40: 610-619.

12 Bartlett NW, Walton RP, Edwards MR, et al. Mouse models of rhinovirus-induced disease and exacerbation of allergic airway inflammation. Nat Med 2008; 14: 199-204.

13 Hewson CA, Haas JJ, Bartlett NW, et al. Rhinovirus induces MUC5AC in a human infection model and in vitro via NF- $\mathrm{kB}$ and EGFR pathways. Eur Respir J 2010; 36: 1425-1435. 